\title{
Anthony Turner, Mathematical Instruments in the Collections of the Bibliothèque nationale de France
}

Londres, BnF/Éditions Brepols, 2018

\section{Catherine Cardinal}

\section{(2) OpenEdition}

\section{Journals}

Édition électronique

URL : http://journals.openedition.org/artefact/5157

DOI : $10.4000 /$ artefact. 5157

ISSN : 2606-9245

\section{Éditeur :}

Association Artefact. Techniques histoire et sciences humaines, Presses universitaires du Midi

\section{Édition imprimée}

Date de publication : 15 décembre 2019

Pagination : 366-369

ISBN : 978-2-8107-0667-9

ISSN : 2273-0753

\section{Référence électronique}

Catherine Cardinal, « Anthony Turner, Mathematical Instruments in the Collections of the Bibliothèque nationale de France ", Artefact [En ligne], 11 | 2019, mis en ligne le 27 novembre 2020, consulté le 30 novembre 2020. URL : http://journals.openedition.org/artefact/5157 ; DOI : https://doi.org/10.4000/ artefact.5157

Ce document a été généré automatiquement le 30 novembre 2020.

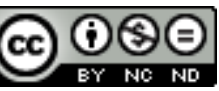

Artefact, Techniques, histoire et sciences humaines est mise à disposition selon les termes de la Licence Creative Commons Attribution - Pas d'Utilisation Commerciale - Pas de Modification 4.0 International. 


\title{
Anthony Turner, Mathematical Instruments in the Collections of the Bibliothèque nationale de France
}

Londres, BnF/Éditions Brepols, 2018

\author{
Catherine Cardinal
}

\section{RÉFÉRENCE}

Anthony Turner, Mathematical Instruments in the Collections of the Bibliothèque nationale de France, Londres, BnF/Éditions Brepols, 2018, 335 p., 260 ill. en couleur.

1 La connaissance des objets conservés à la Bibliothèque nationale de France vient d'être enrichie d'un catalogue qui met en lumière, pour la première fois, la vaste collection d'instruments de mesure, répartie entre le département des Cartes et plans, celui des Monnaies, médailles et antiques et la bibliothèque de l'Arsenal. Établi par Anthony Turner, l'ouvrage présente 138 pièces classées dans onze chapitres. À l'intérieur de chaque catégorie, les objets apparaissent par ordre chronologique. Quelques-uns ont été montrés dans des expositions récentes comme Science et curiosité à la cour de Versailles, au musée national du château de Versailles, Globes. Visions du monde au Louvre Abou Dhabi, transposée en 2019 sous le titre Le monde en sphères, à la BnF/Tolbiac, mais la plupart étaient ignorés ou mal étudiés jusqu'à ce travail. Dans sa préface, Catherine Hofmann, conservateur en chef au département des Cartes et plans, se réjouit des " pépites, parfois découvertes à l'occasion de cet inventaire ».

Une courte introduction sert d'avertissement au lecteur. Les pièces en lien avec la cartographie et la géographie ont été exclues mais les sphères armillaires occidentales ont été sélectionnées parce qu'elles ont un rapport avec les mathématiques en astronomie et astrologie. Les instruments médicaux ont été omis car ils sont l'objet d'une recherche particulière actuellement menée par Muriel Labonnelie. L'ouvrage est avant tout un catalogue descriptif n'ayant pas pour but d'expliquer les usages et 
l'histoire des instruments présentés; le lecteur est invité, pour compléter éventuellement son approche, à se référer aux nombreuses monographies publiées sur les astrolabes, les cadrans solaires, les globes ou les balances. À notre avis, celle de l'auteur du présent catalogue, publiée par Sotheby's en 1987, Early Scientific Instruments. Europe 1400-1800, est particulièrement complète. À l'occasion de l'annonce du plan adopté pour chaque notice (signature, inscription, date, matériaux, dimensions, description, commentaire, provenance, fabricant, exposition, numéro d'inventaire, références), il est rappelé que la bibliographie de l'instrument n'est pas exhaustive mais qu'elle mentionne les références les plus intéressantes.

3 Avant le catalogue proprement dit, une analyse d'une dizaine de pages situe l'ensemble dans le contexte des collections d'instruments appartenant à des bibliothèques. Sobrement intitulée "Mathematical Instruments in Libraries", cette étude fait ressortir les motivations qui ont conduit à l'insertion d'instruments dans des bibliothèques depuis l'époque médiévale et les choix qui ont été faits en fonction des ouvrages scientifiques réunis. L'alliance entre livres et objets apparaît à travers de nombreux témoignages. Ainsi, pour faciliter l'étude des ouvrages grecs et latins sur l'astronomie, Cosme I ${ }^{\text {er }}$ fit construire quatre sphères armillaires, livrées en 1574-1575. Aux châteaux de Fontainebleau et de Blois, à la Bodleian Library, à la bibliothèque de St John's College, à Oxford, le même constat de complémentarité peut être fait. Autre exemple: le cabinet de curiosités créé par l'abbé du Molinet (1620-1687), à l'abbaye Sainte-Geneviève, était conçu pour compléter la collection de livres. Au XvIII siècle, l'adjonction d'instruments à une bibliothèque est encore habituelle dans le milieu des amateurs de sciences. Un des plus célèbres est Charles Boyle, duc d'Orrery (1676-1731), qui légua ses collections, à condition qu'elles restassent réunies au Christ Church College, à Oxford, où il avait étudié.

4 La première section du catalogue est réservée aux astrolabes, au nombre de sept, sans compter une araignée et un astrolabe-quadrant. Pour l'analyse des exemplaires comportant des inscriptions arabes, Anthony Turner s'est appuyé sur la précieuse collaboration de Silke Ackermann, directeur du Musée d'histoire des sciences de l'université d'Oxford et de Taha Yasin Arslan, assistant professeur au Département d'histoire des sciences de l'université Medeniyet, à Istanbul. Les pièces sont méticuleusement décrites, les indications en langue arabe intégralement traduites. L'exceptionnel astrolabe datant $\mathrm{du} \mathrm{x}^{\mathrm{e}}$ siècle, réalisé par Ahmad ibn Khalaf, ayant appartenu à Ja'far, fils du calife abbasside al-Muktatî-'Ilâh', connu pour son goût de l'astronomie, bénéficie ainsi d'une notice de six pages richement illustrées. Le même soin est apporté à la description et à l'illustration d'un astrolabe persan à cinq tympans, daté de 1659-1660, qui a la particularité de mentionner quarante-six localités, remarquablement décoré par Muhammad Mahdî al-Khâdim al-Yazdî. Citons encore deux astrolabes, datés de 1526 et signés par Georg Hartmann (1489-1564), figurant parmi les trois astrolabes les plus anciens de ce mathématicien qui travailla à Nuremberg.

Dans le chapitre suivant, consacré aux neuf sphères célestes de la collection, l'intérêt se porte sur un globe en bronze, daté vers 1080, attribué à Ibrâhî ibn Sa'îd as-Sahlî alWazzân, actif à Tolède et Valence entre 1067 et 1086. Supportée par un haut trépied en bois doré richement orné $(\mathrm{H} .145 \mathrm{~cm})$, la sphère «mouvante" signée par Jérôme Martinot (1671-1724), horloger du Roi, se distingue par sa double qualité d'objet d'art et d'instrument astronomique. Cette sphère armillaire mécanique géocentrée, datée 
entre 1709 et 1718, est la cinquième pièce de l'horloger - la seule conservée - d'une série inaugurée par la sphère mobile présentée à Louis XIV, le 28 février 1701. Parmi les instruments cosmographiques qui forment le troisième chapitre du catalogue, le regard se porte sur le planisphère mécanique montrant le mouvement des étoiles et des planètes, conçu par l'astronome danois ole Roemer (1644-1710) et exécuté par l'horloger Isaac Thuret (1630-1706). La spectaculaire machine fut décrite par Roemer aux membres de l'Académie des sciences en août 1680. Un deuxième planisphère, également réalisé par Thuret mais non signé, destiné à montrer les éclipses, fut présenté par l'astronome dans la même séance. Les deux pièces formant pendant, de mêmes dimensions (H. $98 \mathrm{~cm}$; D. $47 \mathrm{~cm}$ ), similairement décorées de dauphins et des attributs royaux, sont réunies au sein du Département des Cartes et plans.

6 La BnF possède d'étonnants et luxueux instruments à mesurer le temps. Anthony Turner attire en particulier l'attention sur un cadran solaire signé par Pierre Le Maire (1670-1745). De forme carrée ( $42 \mathrm{~cm}$ de côté), la base en marbre blanc porte le cercle des heures et le gnomon. Le centre est occupé par une carte du monde en projection stéréographique - avec Paris comme point central - entourée d'une échelle graduée en heures et minutes. Aux angles, apparaissent les armes de son propriétaire, Michel de Chamillart, seigneur de Montfermeil, ministre de la Guerre entre 1701 et 1709, et de son épouse Louise de Rebours. Anthony Turner a également mis en évidence la rareté d'un calendrier perpétuel signé par Andreas Pleninger à Regensburg, daté 1603. Gravé sur une plaque de mellite, décoré des allégories des éléments et des saisons, il est monté sur une table ornée de motifs en marqueterie $(\mathrm{H} .82,5 \mathrm{~cm})$. La bibliothèque de l'Arsenal peut s'enorgueillir d'une horloge, datée vers 1720 , en ébène et marqueterie de bronze et écaille, ornée de figures en bronze doré représentant les quatre continents. Signée "Julien le Roy» sous le cadran et "Gilles Martinot A Paris » sur la platine du mouvement, la pendule $(\mathrm{H} .125 \mathrm{~cm})$ à sonnerie des heures, demies et quarts, caractérisée par un cadran ovale, est montée sur un piédestal assorti (H.165 cm), orné d'un médaillon figurant Atlas et Hercule. Le modèle attribué à l'ébéniste Gilles-Marie Oppenord (1672-1742) est connu par quatre autres exemplaires (Wallace Collection, Waddesdon Manor, Getty Museum, Detroit Museum of Arts).

7 Outre des descriptions extrêmement précises, accompagnées d'environ quatre cents notes permettant de les approfondir, les notices sont enrichies de commentaires faisant état d'analyses antérieures et proposant de nouvelles approches, d'informations détaillées sur les fabricants et leur production. Les provenances et l'historique des pièces sont développés autant qu'il est possible. Soulignons que le travail de l'auteur est mis en valeur par une présentation graphique claire, un nombre considérable d'illustrations et que sa rédaction, élégante et sobre, permet au lecteur de s'introduire aisément dans ses analyses. En fin de volume, un tableau synoptique proposant un aperçu général des pièces, indiquant leur provenance et leur localisation, accompagné de leur numéro d'inventaire et de catalogue aurait néanmoins facilité la consultation. La numérotation des photographies aurait été également utile. La constatation de ces manques dans l'utilisation de l'ouvrage fait ressortir, par leur caractère dérisoire, l'énormité de la tâche accomplie par Anthony Turner pour mener à terme un projet longuement mûri. L'exposition des pièces les plus remarquables de la collection est prévue dans la galerie du site Richelieu de la BnF, à partir de septembre 2021. 


\section{AUTEURS}

\section{CATHERINE CARDINAL}

Université Clermont-Auvergne/CHEC 